\title{
DUST FORMATION IN WC-STAR SHELLS
}

\author{
E. SEDLMAYR AND H. GASS \\ Institut für Astronomie und Astrophysik \\ Technische Universität Berlin, PN 8-1 \\ Hardenbergstraße 36 \\ D-1000 Berlin 12
}

\begin{abstract}
The conditions of dust formation in the winds of WC-stars are discussed. Simple analytical estimations for the existence of neutral carbon zones where carbon nucleation might occur are given. It is shown that efficient dust formation can take place in the CI region if the effective temperature of the star is below $\sim 25000 \mathrm{~K}$ and the density at the base of the wind is larger than $\sim 10^{11} \mathrm{~cm}^{-3}$.
\end{abstract}

\section{Introduction}

The condensation of solid particles requires conditions where the temperature is cool enough and the density is still sufficiently high for allowing clusters to be formed. Typical values for systems of effective dust formation require a kinetic temperature $T \leq 1200 \mathrm{~K}$ and a density of the nucleating species $n \geq 10^{5} \mathrm{~cm}^{-3}$. These conditions are usually to be met in the outflows of cool giants and supergiants but also at some stages in the expanding shells of novae and supernovae and in the equatorial planes of $\mathrm{B}[\mathrm{e}]$-stars which all are known to be objects of pronounced circumstellar dust formation. Thus, in a first view, it seems to be difficult to understand that the appearance of circumstellar dust formation is not merely confined to these objects where sufficiently cool conditions naturally are expected, but that effective dust formation also occurs in the expanding shells of such high-temperature objects like WR-stars, as is manifested by a pronounced IR excess which has to be attributed to the presence of solid particles forme $d$ in their stellar wind.

Still being far from having a full understanding of this phenomenon, we only want to present some basic ideas which might be important for this complex and, possibly, could shed some light on the problem how grains can be formed under such in principle hostile conditions as encountered in the shells of WC-stars.

This concerns in particluar late type WC'-stars with effective temperatures below $\mathbf{3 0 0 0 0}$ $\mathrm{K}$ where IR-measurements indicate a Planckian emission characteristic which undoubtedly is due to thermal emission of dust grains having an internal temperature around $1000 \mathrm{~K}$. It is the aim of this short contribution to focus on this interesting fact in more detail.

The dust structure of the shells of late WC-stars are determined by

- a hot stellar radiation field: $T_{*} \simeq 20 \ldots 30 \cdot 10^{3} \mathrm{~K}$

- a high (steady) mass loss rate: $\dot{M} \simeq 10^{-5} \ldots 10^{-3} M$. yr 
- a high expansion veloctiy: $v \simeq 1000 \mathrm{~km} / \mathrm{s}$

- a high carbon (and oxygen) abundance: $\epsilon_{C} \gtrsim \epsilon_{O} \gg \epsilon_{H}$

For more details see Maeder and Meynet (1987), Chiosi and Maeder (1986), and Williams et al. (1987). Assuming $R_{*}=10 R_{\ldots}$ as a characteristic value for the stellar radius, the above numbers for the mass loss rate and for the expansion velocity provide a typical number density of the expanding material of the order of $n \simeq 10^{11} \mathrm{~cm}^{-3}$ at $r=R_{*}$.

Except the chemical composition, these parameters basically are not very different from those characterizing a nova outburst where an expanding shell is expelled from the hot central star. (see e.g. Bode and Evans, 1983):

- effective temperature: $8 \cdot 10^{3} \mathrm{~K} \leq T_{*} \lesssim 40 \cdot 10^{3} \mathrm{~K}$

- shell density: $n \simeq 10^{11} \mathrm{~cm}^{-3}$

- expansion velocity: $=2000 \mathrm{~km} / \mathrm{s}$

- abundance: $\epsilon_{H} \gg \epsilon_{C}>\epsilon_{O}$

For the expanding shells of novae, we have investigated the process of dust formation. Theoretical model calculations for the evolution of the shells of novae including time dependent chemistry and the equations for dust formation and grow th indicate that effective grain formation only can occur at those regions where sufficient neutral atoms or molecules of the dust forming species are present. This concerns in particluar the nucleation phase, i.e. the formation of the critical clusters out of the gas phase which, if formed, are able to grow or at least to survive in the expanding medium. A necessary condition for this region is that carbon is predominantly neutral. For this reason, the time required for carbon ionization is decisive for the dust formation problem.

The time scale $\tau_{i o n}$ for a complete ionization of the shell is determined by two parameters: The effective temperature of the central object and the density within the shell. Thus, dust nucleation in a nova shell is controlled by the competition of the characteristic time scale $\tau_{\text {ion }}$ necessary for the carbon ionization of the shell and the time scale $\tau_{\mathrm{d}}$ charactieristic for the formation of macroscopic particles due to gas-kinetic collisions.

If $\tau_{\text {ion }}<\tau_{\mathrm{d}}$, the ionization front overruns the shell and ionizes the dust forming species before effective dust formation can take place. In this case, no dust will form in a nova outburst.

If $\tau_{10 n}>\tau_{\mathrm{d}}$, dust nucleation will take place and the dust formation can even be completed before the shell is ionized.

The question whether in WC-stars the outstreaming matter is ejected in subsequenced shells analoguos to a nova or as a continuos flow like a wind situation is still open. In any case, the above close analogy between a nova shell and the outflow of a WC-star suggests to apply a similar method to investigate dust formation in the outflows of WC-stars.

Both, for novae and WC-stars a spherical symmetric outflow is assumed in our models. Then only two modifications of our novae calculations are necessary for being applied to WC-stars:

i) For a stationary wind of a WC-star, which is the usual assumption in model calculations, one has not to compute the time evolution of the various ionization fronts but only the extension of the corresponding ionization spheres.

ii) The wind material of WC-stars consists mainly of carbon and oxygen (cf. Maeder and Meynet, 1987) and, hence, neutral C, atoms will be the dominating dust forming species. 
For our further investigations, therefore, we assume a continuos spherical symmetric stationary wind of a WC-star consisting mainly of carbon.

\section{The Ionization Structure of a WC-Shell}

According to the above arguments, the dust formation region is situated outside the CII ionization sphere, the extension of which is estimated by the following manner:

The degree of ionization of a pure carbon mixture

$$
f=\frac{n_{\mathrm{CII}}}{n_{\mathrm{CI}}+n_{\mathrm{CII}}}
$$

with $n_{\mathrm{CI}}$ and $n_{\mathrm{CII}}$ being local number density of carbon atoms and ions, respectively, is given by the local balance of ionization and recombination processes expressed by

$$
(1-f)\left(\frac{R_{*}}{r}\right)^{2} \int_{\nu_{0}}^{\infty} \frac{\pi F_{* \nu}}{h \nu} \sigma_{\nu} e^{-\tau_{\nu}} d \nu-\alpha n f^{2}=0,
$$

where $F_{* \nu}$ is the spectral flux of radiation with frequency $\nu$ emerging from the surface of a central object, $\sigma_{\nu}$ the ionization cross section, $\nu_{0}$ the threshold frequency for ionization, $\tau_{\nu}(r)$ the monochromatic optical depth, $\alpha$ the recombination coefficient, $r$ the distance, and $h \nu$ the photon energy. In Eq. (2) is assumed that carbon is only single ionized and, thus, the electron density equals the ion density.

With $n=n_{\mathrm{CI}}+n_{\mathrm{CII}}$ being the total carbon density, $\tau_{0}=\tau_{\nu_{0}}$ the outwards directed optical depth of the shell at $\nu_{0}$ with $\tau_{0}\left(R_{*}\right)=0$ the solution of the quadratic equation (2) is given by

$$
f=\frac{a}{2 b}-\frac{1}{2 c}\left(a^{2}-4 a b\right)^{1 / 2}
$$

where the abbreviations

$$
a\left(\tau_{0}\right)=\left(\frac{R_{*}}{r}\right)^{2} \int_{\nu_{0}}^{\infty} \frac{\pi F_{* \nu}}{h \nu} \sigma_{\nu} e^{-\tau_{\nu}} d \nu \quad ; \quad b\left(\tau_{0}\right)=-\alpha n
$$

have been introduced.

By a Taylor series expansion of Eq. (3) up to the second order and insertion into the definition equation of the $\tau_{0}$-scale

$$
\frac{d r}{d \tau_{0}}=\left(\sigma_{0} n(1-f)\right)^{-1}
$$

with $\sigma_{0}=\sigma_{\nu_{0}}$, one finds

$$
\frac{d r}{d \tau_{0}}=\frac{2 a\left(\tau_{0}\right)}{\sigma_{0} n \alpha n^{2}}
$$

Adopting an inverse square law for the dilution of the initial density $n_{*}$

$$
n=n_{*}\left(\frac{R_{*}}{r}\right)^{2}
$$


and Kramer's rule for the ionization cross section

$$
\sigma_{\nu}=\sigma_{0}\left(\frac{\nu_{0}}{\nu}\right)^{3}
$$

integration of Eq. (6) from $R_{*}$ to a distance $R_{C}$ where the contribution of the ionizing radiation field vanishes, yields for the radius of the CII sphere $R_{C}$ :

$$
R_{C}=\left[1-\frac{2 \int_{\nu_{0}}^{\infty} \frac{\pi F_{* \nu}}{h \nu} d \nu}{\alpha n_{*}^{2} R_{*}}\right]^{-1} R_{*}
$$

It can be seen from Eq. (9) that for radiation fields with $T_{\text {eff }} \lesssim 25000 \mathrm{~K}$ and $R_{*}=$ $10^{12} \mathrm{~cm}$ the existence of a finite carbon ionization sphere depends very sensitively on the initial particle density $n_{*}$. For a Planckian radiation field and $n_{*}=5 \times 10^{12} \mathrm{~cm}^{-3}$, the ionization sphere is $R_{C} \cong R_{*}$ whereas for a particle density $n_{*} \cong 10^{12} \mathrm{~cm}^{-3}$, the ionization sphere is always infinite. For a realistic estimate of the radiation field, the above blackbody radiation field should be modified by a factor $e^{-\tau_{*}}$ where $\tau_{*}$ accounts for the photoionization of carbon inside the stellar atmosphere. In order to account for this effect, we assume $\tau_{*} \sim 5$, which seems to be a reliable value for such type of atmospheres. Introducing this factor into eq. (9) yields always a finite value for $R_{\mathrm{C}}$ for effective temperatures $\leq 25000 \mathrm{~K}$ and $n_{*} \geq 10^{11} \mathrm{~cm}^{-3}$, which is in good agreement with the observational data as provided by Williams et al. (1987) in Table 8.

Outside the CII-sphere, the energy input by photoionization decreases rapidly and the temperature drops abiabatically from the temperature of the CII front, $T_{C}=4000 \mathrm{~K}$, to values where dust formation becomes possible.

\section{Dust condensation}

\subsection{CHEMICAL RESTRICTIONS}

Efficient dust formation generally requires the nucleating species to consist of molecules from abundant elements which are not blocked by high bond energies and allow for high temperature condensates. In case of a carbon star, in particular the following molecules contribute to grain formation: $\mathrm{C}, \mathrm{C}_{2} \mathrm{H}, \mathrm{C}_{2} \mathrm{H}_{2}, \mathrm{SiC}$. For a WC,-star, due to a lack of hydrogen, carbon-hydrogen molecules are not present. Hence, the only nucleating species left are $\mathrm{C}$ and $\mathrm{SiC}$. As no data of $\mathrm{SiC}$ are abvailable to describe the chemistry of SiC-cluster formation, we confine our calculation on the formation of pure C-clusters. Therefore, our results yield only a lower limit for the condensated material expected.

The above assumption of carbon grain formation is based on the fact that all oxygen should be blocked by the $\mathrm{CO}$ molecule. This is certainly true in case of C-stars where only expansion velocities of the order $\sim 10 \mathrm{~km} \mathrm{~s}^{-1}$ and only moderate UV radiation fields are observed. In case of hot objects like novae and WC-stars, however, this may not always be true because the reaction channels for effective $C O$ formation could be destroyed. As $\mathrm{CO}$ formation then has to proceed via rather slow channels, the high expansion velocity of novae and WC-stars may result in an incomplete $\mathrm{CO}$ blocking and, thus, in a larger amount of available condensable material. 


\subsection{DUST FORMATION AND GROWTH}

From the arguments and estimations in chapter 2 can be seen that a neutral carbon zone where dust condensation may occur can exist only for stars with effective temperatures $T \lessgtr 25000 \mathrm{~K}$ and initial densities $n_{*} \gtrsim 10^{11} \mathrm{~cm}^{-3}$. To investigate dust formation in the neutral carbon zone, we assume an adiabatic temperature $T$ :

$$
T=T_{\mathrm{C}}\left(\frac{R_{\mathrm{C}}}{r}\right)^{4 / 3}
$$

and the density structure of eq. (7):

$$
n=n_{\mathrm{C}}\left(\frac{R_{\mathrm{C}}}{r}\right)^{2}
$$

with $n_{\mathrm{C}}$ the carbon density at the inner edge of the CI-region.

With the value for $T_{\mathrm{C}}=4000 \mathrm{~K}$ given in chapter 2 and $n_{\mathrm{C}}=10^{11} \mathrm{~cm}^{-3}$, for instance, the supersaturation ratio of carbon at $2 R_{\mathrm{C}}$ is $10^{10}$. At this radius the gas temperature has decreased to $1600 \mathrm{~K}$.

Applying classical homogeneous nucleation theory (Gail, Seldmayr, 1988) one finds that for suitably choosen parameters dust formation is possible within a region $R_{\mathrm{C}} \leq r \leq 1000 R_{*}$. The results indicate that the nucleation rates $J$ are in the range $10^{\circ} \leq J \leq 10^{9} \mathrm{~cm}^{-3} \mathrm{~s}^{-1}$. Thus, the dust formation process can start very rapidly at a well defined radius. This is in agreement with observed energy distributions (Williams et al., 1987). Due to the large consumption of condensating material, the nucleation ceases already after some $10^{4} \mathrm{~s}$. The short time for growth allows only the formation of rather small grains. The pertinent results should not be extremely different from those in nova shells, where a mean particle size of 40 monomeres has been found.

Estimating the radii of the inner edge of the dust spheres and the dust densities, the expected results may well fit with the observational data provided by Williams et al. (1987).

\section{Conclusions}

The above arguments confirm that dust formation quite naturally might occur in the environments of WC-stars with $T_{\text {eff }} \lesssim 25000 \mathrm{~K}$ and densities $n_{*} \gtrsim 10^{11} \mathrm{~cm}^{-3}$. Further quantitative informations concerning the detailed structure of the temperature, the ionization, the dust density and the grain charcteristics in the wind, however, have to be based on more elaborate model calculations.

\section{References}

Bode, M.F., Evans, A.: 1983, Quart. J. Roy. astr. Soc. 24, 83

Chiosi, C., Maeder, A.: 1986, Ann. Rev. Astron. Astrophys. 24, 329

Gail, H.-P., Sedlmayr, E.: 1988, Astron. Astrophys. 206, 153

Maeder, A., Meynet, G.: 1987, Astron. Astrophys. 182, 243

Williams, P.M., van der Hucht, K.A., The, P.S.: 1987, Astron. Astrophys. 182, 91 


\section{DISCUSSION}

Cassinelli: (1) In the model of Zickgraf of $\mathrm{B}[\mathrm{e}]$ stars, he proposes that the dust formation forms in a disk in the equatorial zone. Is there any reason that you might think this is the case ? (2) Do you think that is the case for WC stars also?

Sedlmayr: This is true, we have done such calculations. If you calculate the radiative transfer, if you take a two component-model with a hot polar wind and a slow equatorial wind with a dense cool equatorial shell, then you can easily arrive at conditions of disk formation. (2) If I take my nova results, they have conditions identical to the WC stars outside the CII regions; we have the same density, we have the same UV field, say, a UV field of $20000 \mathrm{~K}-25000 \mathrm{~K}$, we have the same expansion velocity, and therefore the timescales. Therefore I would guess the same results, of course within an order of magnitude, as we have for novae. I think that dust formation for WC stars is very natural.

Cohen: I think that you can test directly for the presence of dusty disks in WC9 stars by optical polarization. In "the old days", Gary Schmidt and I and Len Kuhi observed some WC9's with spectropolarimetry. We found substantial polarization compared with other WR's, but we felt it was merely interstellar in origin.

Sedlmayr: As I wanted to point out, in our opinion dust formation in winds of late type WC stars resembles very much that of a nova and should occur outside the $C I I$ region in a sufficiently dense shell. Disk like dust formation we expect for $B[e]$ stars. 